\title{
Mass Balance Reconciliation for Bilinear Systems: A Case Study of a Raw Mill Separator in a Typical Moroccan Cement Plant
}

\author{
S. Fellaou \\ Laboratory for Analysis and Synthesis of Industrial Processes \\ (LASPI), University Mohammed V Agdal Rabat, \\ Mohammadia School of Engineers, Rabat, Morocco \\ Soukaina.fellaou@research.emi.ac.ma
}

\author{
T. Bounahmidi \\ Laboratory for Analysis and Synthesis of Industrial Processes \\ (LASPI), University Mohammed V Agdal Rabat, Morocco \\ and Euro-Mediterranean University of Fez, Fez, Morocco \\ tbounahmidi@gmail.com
}

\begin{abstract}
Stream flow rates and their several compositions are measured in a typical cement raw mill separator. In order to simultaneously reconcile flow and composition measurements in this circuit, the component mass balances was included as constraints which contain the products of flow rate and composition variables in the data reconciliation problem. In this paper, the effectiveness of simultaneous procedures for bilinear data reconciliation is established, the numerical problem constraints were coded in MATLAB and a mass balance model is built. Moreover, based on the difference between the measured and reconciled data it was found that it performs optimally.
\end{abstract}

Keywords-mass balance; data reconciliation; bilinear system; cement industry.

\section{INTRODUCTION}

Process variables are measured for control purposes and also for economic evaluation and optimization purposes. The estimation of true conditions of process states can impact in different ways to the efficiency of the process. From a statistical point of view, the method that adequately supports data quality improvement is data reconciliation, which can be employed to adjust the entire data set to fit the system constraints [1] i.e. to fulfill the process model. Nowadays, the data reconciliation technique has been applied to many different industrial areas such as mineral industry processes, chemical production plants, and nuclear power plants [2]. However it has not yet been commonly used in the Moroccan industry. The purpose of this paper is to gather a full understanding of how data reconciliation can be investigated in a satisfactory manner for cement manufacturing process performance evaluation, to present what the most likely state of the system is and to verify the acceptability and the accuracy of measurements. In a processing plant, the total mass entering and leaving any portion of it must obey the mass conservation constraints. Since these constraints covered the case of multicomponent balances, the term bilinear data reconciliation is consequently used. This procedure contributes to the improvement of the process data quality which is the basis for process control, production accounting goals, as well as optimization.
A vast amount of literature is available in the field of data reconciliation. In 1961, Kuehn and Davidson [3] were the first to publish an analysis of data reconciliation in the process industry. They solved a linear data reconciliation problem where the process was in steady state with all variables measured. A subsequent publication [4] defined the concepts of observability and redundancy and worked out many of the basic ideas in the field. In [5] it was found that the base method requires a process system in steady state conditions. In recent years, many industrial application of data reconciliation are related in scientific papers involving various processing industries [6-11]. In the same vein, sufficient breadth in software packages supporting data reconciliation are available and allows to manage the operations of different processes (Datacon, Sigmafine, Vali, Recon, Ebsilon Validate, Datrec, Dval, Inlibra).

\section{MOTIVATION AND PROBLEM STATEMENT}

The cement industry is responsible for around 33.3 per cent of industrial energy consumption in Morocco. It has been remarked as an intensive consumer of natural raw materials, fossil fuels and a major source of pollutants as well. Thus, evaluating and maintaining the manufacturing process close to an optimum performance in this industry is has become a necessity. With the purpose of evaluating their performance and determining current efficiencies, balances for cement process must be performed and this cannot be achieved unless the process is first defined and measured. The primary focus of process evaluation is usually equipment specification. In fact, the question of sufficiency of information to make the material balance is answered by the degree of freedom analysis [12]. Thus, to make sure that the number of variables and equations are in balance prior to attempting optimal solution. After fixing the degree of required knowledge of the process, the balances which are defined by the measured data, should have been extensive enough to more than satisfy the balance equations [13]. However, due to random and systematic errors, most plant systems face information redundancy in the measurement, in the sense that the system amount of available data exceeds the minimum amount necessary for a unique determination of the independent variables that determine the plant model 
which yield a negative value for the number of degrees of freedom.

Once the process constraints are created, all available or redundant measured variables must first be assigned to the model streams and units together with their respective measurement uncertainties [14]. Additionally, the key variables in a cement processing unit are usually flow rates and material chemical compositions. Their reconciliation with the laws of mass conservation throughout the system is essential and can be used to ensure consistent product quality.

The applicability of the data reconciliation approach is confirmed through an industrial case study of a redundant typical mill/separator circuit, as shown in Figure 1. The process for raw meal production requires tube mill as the main machine for grinding and a separator for splitting the material from the grinding mill (F1) into a fine (F3) and coarse fraction (F2), the corresponding measurements data $Y_{i}$ is given in Table I. Material input are limestone, silica stone, clay, cooper slag and the product (F3) is raw meal.

Material balances are fundamental to the control of processing, particularly in the control of yields of the products. Equilibrium of the material balance equations consists of estimating the true quantities that they should be consistent. The process studied has one inlet stream and two outlet streams. Initially, mass flow rate and composition of every stream are assumed to be directly measured. According to the law of conservation of matter for the circuit studied, the mass going into the separator must balance with mass coming out. The measurement values are assumed to give an image of the process steady-state behavior. Hence, the constraints of mass conservation are written in the form:

$$
\begin{gathered}
\mathrm{f}_{1,1}+\mathrm{f}_{2,1}-\mathrm{f}_{3,1}=0 \\
(\ldots) \\
\mathrm{f}_{1,11}+\mathrm{f}_{2,11}-\mathrm{f}_{3,11}=0 \\
\mathrm{f}_{\mathrm{i}, \mathrm{j}}=\mathrm{F}_{\mathrm{j}} \mathrm{w}_{\mathrm{i}, \mathrm{j}}
\end{gathered}
$$

Where $F_{i}$ is the vector of the measured flow rates and $W_{i, j}$ are the composition vectors. The verification whether measured data (given in Table I) satisfy the balance equation, indicates that the above equation is not fulfilled. This means that the material input and output of the unit do not balance,

\begin{tabular}{|c|c|c|c|c|c|c|c|c|}
\hline $\begin{array}{c}\text { Stream } \\
\text { components }\end{array}$ & Variables & $\begin{array}{c}\text { Raw } \\
\text { Measurement } \\
Y_{1}(\mathrm{~kg} / \mathrm{h})\end{array}$ & $\begin{array}{c}\text { Standard } \\
\text { deviation of } \\
\text { measured value } \\
(\mathrm{kg} / \mathrm{h})\end{array}$ & $Y_{1}^{\prime \prime}-3 \sigma$ & $\begin{array}{c}\text { Reconciled } \\
\text { Measurement } Y_{i}^{*} \\
(\mathbf{k g} / \mathbf{h})\end{array}$ & $\mathrm{Y}_{1}^{*}+3 \sigma$ & $\begin{array}{c}\text { Standard } \\
\text { deviation of } \\
\text { reconciled value } \\
(\mathrm{kg} / \mathrm{h})\end{array}$ & $\begin{array}{c}\text { Adjustment } \\
(\mathrm{kg} / \mathrm{h})\end{array}$ \\
\hline Total feed & $\mathrm{F}_{1}$ & 300940 & 140 & 300520 & 300530 & 301360 & 43 & -410 \\
\hline $\mathrm{SiO2}$ & $\mathrm{W}_{1,1}$ & 0,135 & 0,014 & 0,093 & 0,1467 & 0,177 & 0,0102 & 0,0117 \\
\hline Al2O3 & $\mathrm{W}_{1,2}$ & 0,033 & 0,009 & 0,006 & 0,0350 & 0,06 & 0,0018 & 0,0020 \\
\hline Fe2O3 & $\mathrm{W}_{1,3}$ & 0,0211 & 0,0015 & 0,0166 & 0,0213 & 0,0256 & 0,0011 & 0,0002 \\
\hline $\mathrm{CaO}$ & $\mathrm{W}_{1,4}$ & 0,4343 & 0,0249 & 0,3596 & 0,4705 & 0,509 & 0,0104 & 0,0362 \\
\hline MgO & $\mathrm{W}_{1,5}$ & 0,0125 & 0,0006 & 0,0107 & 0,0125 & 0,0143 & 0,0006 & 0,0000 \\
\hline SO3 & $\mathrm{W}_{1,6}$ & 0,0016 & 0,0002 & 0,001 & 0,0017 & 0,0022 & 0,0002 & 0,0001 \\
\hline K2O & $\mathrm{W}_{1,7}$ & 0,0052 & 0,0007 & 0,0031 & 0,0051 & 0,0073 & 0,0004 & $-0,0001$ \\
\hline TiO2 & $\mathrm{W}_{1,8}$ & 0,0022 & 0,0003 & 0,0013 & 0,0023 & 0,0031 & 0,0002 & 0,0001 \\
\hline P2O5 & $\mathrm{W}_{1,9}$ & 0,0016 & 0,0002 & 0,001 & 0,0016 & 0,0022 & 0,0002 & 0,0000 \\
\hline MnO & $\mathrm{W}_{1,10}$ & 0,0007 & 0,0001 & 0,0004 & 0,0007 & 0,001 & 0,0001 & 0,0000 \\
\hline LOI & $\mathrm{W}_{1,11}$ & 0,3528 & 0,0056 & 0,336 & 0,3518 & 0,3696 & 0,0000 & $-0,0010$ \\
\hline $\begin{array}{c}\text { Separator } \\
\text { rejects }\end{array}$ & $\mathrm{F}_{2}$ & 170290 & 37 & 170179 & 170320 & 170401 & 36 & 30 \\
\hline SiO2 & $\mathrm{W}_{2,1}$ & 0,137 & 0,038 & 0,023 & 0,1498 & 0,251 & 0,0189 & 0,0128 \\
\hline Al2O3 & $\mathrm{W}_{2,2}$ & 0,035 & 0,001 & 0,032 & 0,0350 & 0,038 & 0,0010 & 0,0000 \\
\hline Fe2O3 & $\mathrm{W}_{2,3}$ & 0,0213 & 0,0019 & 0,0156 & 0,0210 & 0,027 & 0,0016 & $-0,0003$ \\
\hline $\mathrm{CaO}$ & $\mathrm{W}_{2,4}$ & 0,4338 & 0,0576 & 0,261 & 0,4661 & 0,6066 & 0,0190 & 0,0323 \\
\hline MgO & $\mathrm{W}_{2,5}$ & 0,0121 & 0,003 & 0,0031 & 0,0124 & 0,0211 & 0,0017 & 0,0003 \\
\hline SO3 & $\mathrm{W}_{2,6}$ & 0,0057 & 0,0016 & 0,0009 & 0,0005 & 0,0105 & 0,0007 & $-0,0052$ \\
\hline K2O & $\mathrm{W}_{2,7}$ & 0,005 & 0,0008 & 0,0026 & 0,0051 & 0,0074 & 0,0007 & 0,0001 \\
\hline TiO2 & $\mathrm{W}_{2,8}$ & 0,0024 & 0,0003 & 0,0015 & 0,0024 & 0,0033 & 0,0003 & 0,0000 \\
\hline P2O5 & $\mathrm{W}_{2,9}$ & 0,0016 & 0,0003 & 0,0007 & 0,0016 & 0,0025 & 0,0003 & 0,0000 \\
\hline $\mathrm{MnO}$ & $\mathrm{W}_{2,10}$ & 0,0005 & 0,0001 & 0,0002 & 0,0005 & 0,0008 & 0,0001 & 0,0000 \\
\hline LOI & $\mathrm{W}_{2,11}$ & 0,3516 & 0,0229 & 0,2829 & 0,3517 & 0,4203 & 0,0000 & 0,0001 \\
\hline Final product & $\mathrm{F}_{3}$ & 130200 & 26 & 130122 & 130210 & 130278 & 26 & 10 \\
\hline SiO2 & $\mathrm{W}_{3,1}$ & 0,136 & 0,013 & 0,097 & 0,1427 & 0,175 & 0,0118 & 0,0067 \\
\hline Al2O3 & $\mathrm{W}_{3,2}$ & 0,034 & 0,004 & 0,022 & 0,0349 & 0,046 & 0,0039 & 0,0009 \\
\hline Fe2O3 & $\mathrm{W}_{3,3}$ & 0,0224 & 0,0024 & 0,0152 & 0,0218 & 0,0296 & 0,0021 & $-0,0006$ \\
\hline $\mathrm{CaO}$ & $\mathrm{W}_{3,4}$ & 0,432 & 0,033 & 0,333 & 0,4762 & 0,531 & 0,0125 & 0,0442 \\
\hline MgO & $\mathrm{W}_{3,5}$ & 0,0123 & 0,0024 & 0,0051 & 0,0126 & 0,0195 & 0,0021 & 0,0003 \\
\hline SO3 & $\mathrm{W}_{3,6}$ & 0,005 & 0,001 & 0,002 & 0,0034 & 0,008 & 0,0009 & $-0,0016$ \\
\hline K2O & $\mathrm{W}_{3,7}$ & 0,0052 & 0,0006 & 0,0034 & 0,0052 & 0,007 & 0,0006 & 0,0000 \\
\hline TiO2 & $\mathrm{W}_{3,8}$ & 0,0022 & 0,0004 & 0,001 & 0,0022 & 0,0034 & 0,0004 & 0,0000 \\
\hline P2O5 & $\mathrm{W}_{3,9}$ & 0,0015 & 0,0004 & 0,0003 & 0,0015 & 0,0027 & 0,0003 & 0,0000 \\
\hline $\mathrm{MnO}$ & $\mathrm{W}_{3,10}$ & 0,0008 & 0,0002 & 0,0002 & 0,0009 & 0,0014 & 0,0002 & 0,0001 \\
\hline LOI & $\mathrm{W}_{3,11}$ & 0,3519 & 0,0112 & 0,3183 & 0,352 & 0,3855 & 0,0000 & 0,0001 \\
\hline
\end{tabular}
so data reconciliation is necessary to derive estimates of the flows that satisfy the above flow balances.

TABLE I. BILINEAR DATA RECONCILIATION RESULTS OF RAW MILL/SEPARATOR CIRCUIT 


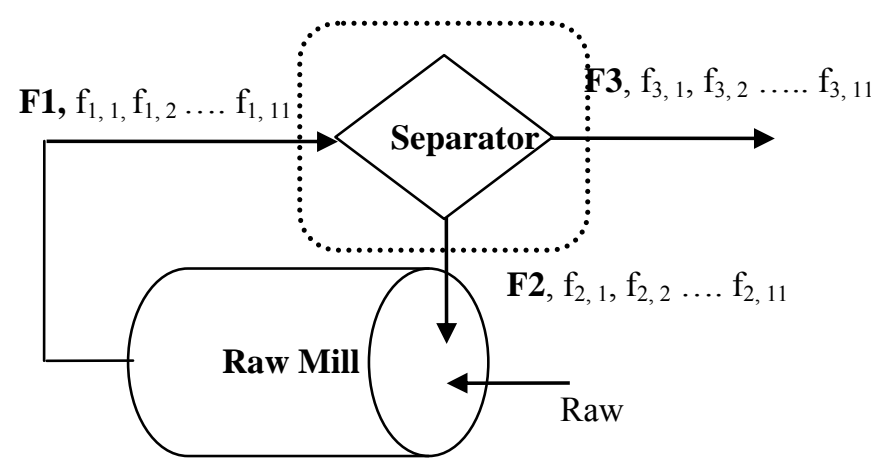

Fig. 1. Schematic diagram of a typical cement raw mill/separator circuit

\section{DATA RECONCILIATION FOR BILINEAR SYSTEMS}

\section{A. Steady-state linear data reconciliation}

Steady-state mass balance process variables are related by mass conservation laws. Although in the presence of measurement errors the balance equations are not satisfied exactly [16]. In such cases the number of balance equations in the system is greater than the number of unknown variables to calculate, some system measurements are then said to be redundant. Moreover, it is highly improbable that data will be fully consistent from the viewpoint material balance since they are by definition individual normally distributed around a mean value. In other words, it is impossible to calculate the unknown so that the material balances are respected. In this context, for measurement adjustment, data reconciliation technique applies a correction to each measurement to force the estimated values to exactly obey the balance equations. In other words, it is devoted to determine the most likely process state with the smallest possible uncertainties. In this paper, the redundant measurements were exploited through the Lagrange multiplier method to improve the quality of available measures so that all the mass balance constraints are met simultaneously by the adjusted values. The reconciliation problem is thus a constrained optimization problem with the objective function given by :

$$
\text { Minimize } Y_{i}^{*}\left[\sum_{i} \frac{\left(Y_{i}^{*}-Y_{i}\right)^{2}}{\sigma_{i}^{2}}\right]
$$

$$
\text { Subject to } \mathrm{AY}_{\mathrm{i}}^{*}=0
$$

The parameters involved in bilinear data reconciliation are: $\sigma_{i}^{2}$ the variance associated with the measurement, $Y_{i}$ the vector of measurements (flow rate and composition variables), $\mathrm{Y}_{\mathrm{i}}^{*}$ the vector of reconciled measurement (flow rate and composition variables), and $\mathrm{A}$ represents the incidence matrix. The standard deviation of the measurements plays an essential role in the data reconciliation problem. An estimate of standard deviation can be obtained by using a simple standard deviation based on the number of measurements taken. The formula for the standard deviation $\sigma$ of a measurement $\mathrm{Y}$ is given by

$$
\sigma=\left(\frac{1}{N-1} \sum_{i=1}^{N}\left(Y_{i}-\bar{Y}\right)^{2}\right)^{\frac{1}{2}}
$$

where $\sigma$ is the estimated value of standard deviation, $\mathrm{Y}_{\mathrm{i}}$ is the ith measurement and $\overline{\mathrm{Y}}$ is the arithmetic average of $\mathrm{N}$ observations of the same variable.

The analytical solution (1) is expressed explicitly [15] as:

$$
\mathrm{Y}_{\mathrm{i}}^{*}=\left(\mathrm{I}^{-V A^{\mathrm{T}}}\left(\mathrm{AVA}^{\mathrm{T}}\right)^{-1} \mathrm{~A}\right) \mathrm{Y}_{\mathrm{i}}
$$

where $\mathrm{V}$ is the variance matrix for each of the measurements that contain information about the correlation between them. The constraint matrix, A, is obtained based on the process connectivity information. Furthermore, the data reconciliation procedure for the adjustment of the measurements to close the bilinear system constraints is reduced to a linear data reconciliation problem and the quadratic function (4) calculation is done using MATLAB to give the reconciled values of the inlet and outlet mass flow rate and composition of every stream which are more correctly reflect the actual current performance of the unit than those obtained just from raw measurements.

\section{B. Accuracy indication}

This section covers the verifications actions to take after the reconciliation process to ensure that the reconciled values lie in an acceptable range. Specifically, the accuracy of the measured datum is expressed in terms of the confidence level of its uncertainty [13]. Which indicate how the measurements are adjusted. In bilinear constraint cases in which the objective is to simultaneously reconcile flow rates and compositions measurements, such as the ones presented here, with real industrial data, the level of uncertainty related to the stream component flow rates is compared with a quality indicator to evaluate the overall consistency of the measurements and the reliability of each individual reconciled measurement. The comparison of each of these estimated values with the coverage factor at the prescribed level of confidence are analyzed to determine if the resulting estimates fall within a confidence interval, say within $Y_{i}^{*} \pm U_{i}$ interval $(99,7 \%$ confidence interval). For $99,7 \%$ confidence the coverage factor is 3 and $U_{i}$ can be expressed in terms of $\sigma_{i}$ as follows :

$$
\mathrm{U}_{\mathrm{i}}=3 \sigma_{\mathrm{i}}
$$

Table I shows the balance in each node before and after reconciliation as well as the standard deviation of measurements adjustments for every variable. The comparison of the standard deviations of the raw measurements and reconciled values of the circuit show that the reconciled data is more accurate than the raw data. In addition to this, from the same table, we can observe that the measurement adjustments for all the flows fall in the confidence interval and a drastic improvement of the process performance evaluation is usually brought on by the reconciled data.

\section{RESULTS AND DISCUSSION}

In this paper, the process of data reconciliation is discussed, and a procedure is suggested regarding how to obtain reliable data sets in an efficient way. The measurements of the feed, separator rejects, and product, 
along with their compositions as well as reconciled values are investigated. When using reconciled values, it can be easily verified that while the measured values do not satisfy the flow balances, the reconciled flows satisfy them. Moreover, the results presented for the application of the bilinear data reconciliation method to the typical cement raw mill/separator circuit showed that the proposed technique slightly improves the quality of flow data and the differences between the measured and estimated flows, also referred to as adjustments, is performed optimally. Also, the current operated point is within the normal operating area and assigns a quality to each individual measurement.

\section{CONCLUSION}

In order to assess the validity of the mass balance of the unit studied, data adjustment is accomplished through bilinear data reconciliation technique and contributed to obtain statistically consistent data and estimate operating criteria. The result obtained has an important practical significance as it allows reconciled variables to be calculated with consistently high precision and provides consistent variables that can be used to determine the typical mill/separator circuit with the objective of calculating its efficiency and improved driving system.

\section{ACKNOWLEDGEMENTS}

The authors gratefully acknowledge the support of engineers of the selected cement factory for providing the necessary facilities support to undertake the above work.

\section{REFERENCES}

[1] A. Spindler, "Structural redundancy of data from wastewater treatment systems. Determination of individual balance equations", Water Res., Vol. 57, pp. 193-201, 2014

[2] C. Karlsson, Tools for reconciliation of measurement data for processes at steady-state, Mälardalen University, 2004

[3] H. Davidson, D. R. Kuehn, "Computer control II: mathematics of control”, Chem. Eng. Prog., Vol. 57, No. 6, pp. 1653-1672, 1961

[4] V. Václavek, Studies on System Engineering. III: Optimal Choice of the Balance Measurements in Complicated Chemical Systems, Chemical Engineering Science, Vol. 24, No. 6, pp. 947-955, 1969

[5] R. S. H. Mah, Chemical process structures and information flows, Elsevier, 1990

[6] H. S. Yi, H. Shin, J. H. Kim, C. Han, "Industrial application of gross error estimation and data reconciliation to by production gases in iron and steel making plants", in International Conference on Control, Automation and Systems, Muju Resort, Jeonbuk, Korea, 2001

[7] M. J. Bagajewicz, E. Cabrera, "Data Reconciliation in Gas Pipeline Systems", Ind. Eng. Chem. Res., Vol. 42, No. 22, pp. 5596-5606, 2003

[8] D. Özyurt, R. Pike, "Theory and practice of simultaneous data reconciliation and gross error detection for chemical processes", Comput. Chem. Eng., Vol. 28, pp. 381-402, 2004

[9] M . Schladt, B . Hu, "Soft-sensors Based on Nonlinear Steady-State Data Reconciliation in the Process Industry", Chem. Eng. Process., Vol. 46, No. 11, pp. 1107-1115, 2007

[10] M. F. Martins, C. Amaro, L. S. Souza, R. Kalid, A. Kiperstok, "New objective function for data reconciliation in water balance from industrial processes", J. Clean. Prod., Vol. 18, No. 12, pp. 1184-1189, 2010

[11] X. Jiang, P. Liu, Z. Li, "A Data Reconciliation Based Approach to Accuracy,” Vol. 35, No. 2003, pp. 1213-1218, 2013.

[12] A. E. Morris, G. Geiger, H. Alan, Handbook on material and energy balance calculations in materials processing third edition, NJ, USA: John Wiley \& Sons, Inc, 2011

[13] C. Maurice. Fuerstenau and N. Kenneth and Han, Principles of Mineral Processing. USA, 2009
[14] M. Langenstein, J. Jansky, "Process data reconciliation in nuclear power plants", Process data, Vol. 2, pp. 1-9, 2003

[15] J. Ragot, M. Aubrun, M. Darrouach, "Equilibrage de Bilan matière méthodes et exemples minéralurgiques", Control Comput., Vol. 11, pp. 50-59, 1983

[16] J. Romagnoli, M. C. Sánchez, Data processing and reconciliation for chemical process operations, Academic Press, San Diego, 2000 